\title{
Zur poietischen Funktion von Analogien und Metaphern im interdiskursiven Blickpunkt der Transplantationsmedizin
}

\section{ROMAN MIKULÁŠ}

DOI: https://doi.org/10.31577/WLS.2021.13.4.10

Aus dem ursprünglich in der Zeitschrift Merkur publizierten Aufsatz „Für neue Leben“, in dem der Autor David Wagner seine eigenen Erfahrungen mit der Lebertransplantation schildert, entsteht ein umfassenderer Text, der als Roman mit dem Titel Leben mit dem Preis der Leipziger Buchmesse von 2013 bedacht wurde. ${ }^{*}$ Uns interessiert der Text aus folgenden Gründen: Das Buch ist als Roman angelegt, genauer als Autopathographie, die einen persönlichen und authentisch-reflektierenden Blick auf das Erleben der eigenen Erkrankung richtet und den medizinischen Eingriff, die chirurgische Verpflanzung einer neuen Leber, wie auch die damit zusammenhängenden Erfahrungen schildert. Der medizinische Fachdiskurs spielt dabei eine wesentliche Rolle und er greift stark in die Erzählinhalte hinein. Medizinisches Wissen und persönliche Spiegelungen überlappen einander wie jene teilweise autonomen Sinnbezirke, aus denen oft Analogien hervorgehen, in denen Metaphern bekanntlich ihren Ursprung haben.

Im Folgenden geht es darum, jene Bereiche (Domänen) freizulegen, die zu Analogien zusammengeschlossen werden, um ihre kommunikative Funktion anhand derer eventuellen Umsetzung in Form von Metaphern (wahlweise bekannt auch als between-domain analogies) zu beschreiben. Unter besonderer Berücksichtigung steht dabei die Rolle des medizinischen Spezialdiskurses und die Frage, wie dieser die Analogisierung und Metaphorisierung des eigenen Krankheitserlebens und das Erleben der Organverpflanzung prägt. Dabei stützen wir uns weitgehend auf das analytische Modell aus der Grundlegung einer Theorie der bildlichen Rede von Hans Georg Coenen.

\section{ANALOGIEN UND METAPHERN IN DER ERLEBNISANALYSE}

Auf der metatheoretischen Ebene gehen wir davon aus, dass sich (nicht nur metaphorische) Wirklichkeitskonstruktionen auf Analogien gründen. Hier werden Einsichten von Karl Bühler und Hans Georg Coenen in entsprechenden Zusammenhängen aufgegriffen. Coenen bringt den Grundgedanken klar auf den Punkt: „Nicht jede Analogie begründet eine Metapher, aber jede Metapher setzt eine Analogie voraus“ $(2002,97)$. Es ist nicht die Problemstellung dieses Aufsatzes, auf den zum Teil schwer

* Dieser Beitrag entstand im Rahmen des Projektes VEGA 2/0111/20 „The Interdiscursive Construction of Reality in Literature". 
nachvollziehbaren und oft äquivoken Gebrauch des Analogiebegriffs in den einschlägigen Spezialdiskursen en détail einzugehen und womöglich noch eine klärende Antwort auf die Frage nach dem Zusammenhang zwischen Analogien und Metaphern $\mathrm{zu}$ geben. Es wird stattdessen auf die Theorie der bildlichen Rede von Hans Georg Coenen Bezug genommen, um auf einige Schwierigkeiten mit dem Analogiebegriff in der Metaphernforschung aufmerksam zu machen und diesen für Untersuchungen an literarischen Werken fruchtbar zu machen.

Entscheidend ist die aus der Aristotelischen Metaphernauffassung herrührende Idee davon, dass Metaphern Analogien voraussetzen. Dabei ist es wichtig, dass Coenen jeder Metapher eine analogische Tiefenstruktur unterlegt. Darüber hinaus kann in einem Text „eine Vielzahl von Bildern und Metaphern aus einer kleinen Zahl von Analogiewurzeln hervorwachsen" (114). Hier spricht Coenen von wurzelgleichen Metaphern, die eng miteinander verwoben sind und deren klassenbildende Gemeinsamkeit ihrer Gegenstandsmengen ein Beziehungsnetz bildet. Die diesen Metaphern zugrundeliegende Analogiewurzel (auszuformulieren als Wurzelprädikat) regelt, wie diese Metaphern interpretierbar sind. Sie gehört oft zum Wissensbzw. Erfahrungsfundus eines einigermaßen gut konturierten Kommunikationssystems.

Analogie bedeutet bekanntlich eine Art der Inbezugsetzung: „Die Relation der Analogie besteht zwischen zwei Gegenständen genau dann, wenn ein Beschreibungsinhalt als für beide geltend beansprucht oder anerkannt wird“ (2). Des Weiteren heißt es: „Ein Analogieverhältnis kann explizit markiert [ausdrücklich benannt, R. M]. oder durch Metapherngebrauch präsupponiert werden“" (122). Auch Karl Bühler erkannte die analogische Basis der bildlichen Sprache und erklärte, dass es in der Prozessierung der metaphorischen Bedeutung zu einer selektiven Wirkung der Sphärendeckung kommt. Alle nicht passenden Eigenschaften werden dabei ausgeblendet (abgedeckt). Und er fügt noch hinzu: „Wie solches Abdecken zustande kommt im psychophysischen System, ist eine der zentralen Fragen an die Sprachpsychologie" ([1934] 1965, 349). Bühlers Metapherntheorie ist insofern von Relevanz, da sie sich explizit auf Wilhelm Stählins Erlebnisanalysen bezieht, der in seiner Studie „Zur Psychologie und Statistik der Metaphern“ aus dem Jahr 1914 evident gemacht hatte, dass und in welcher Art Metaphern für die Erlebnisanalyse ${ }^{1}$ entscheidend sein können. ${ }^{2}$

Metaphern zeigen nicht nur Stellen im Konzeptgeflecht unserer Sprachen an, die nicht besetzt sind, sondern sie sagen auch etwas über die Unbrauchbarkeit der existierenden tradierten Konzepte in konkreten Erfahrungssituationen aus. In beiden Fällen handelt es sich nach Bühler - unter Verweis auf Hermann Paul ${ }^{3}$ - um Momente der Ausdrucksnot und der Ausdrucksdrastik. Neben der Ausdrucksnot und der Ausdruckdrastik führt Bühler noch eine weitere, nicht minder wichtige Funktion der Metapher ins Treffen: Es ist die verhüllende Funktion, wie sie von Heinz Werner in seiner Abhandlung Die Ursprünge der Metapher aus dem Jahr 1919 ausgeführt wird. Nach Werner, so Bühler, „entsprang die echte Metapher einmalig aus dem Geiste des tabu und dient nicht dem Hervorheben, sondern einem Verhüllungsbedürfnis" ([1934] 1965, 351). 
Bühlers Versuche, die Metapher in ihrer Funktionalität zu erfassen, stellen einen wichtigen Aspekt unserer Überlegungen dar. Er macht klar, dass es um die Beziehung des Individuums zu seinem Umfeld gehen wird und dass die Verquickung von Kognition und Kommunikation in ihrer vorausgesetzten strukturellen Kopplung ins Auge gefasst werden soll.

\section{GRUNDLEGUNG EINER THEORIE DER BILDLICHEN REDE - EINE HERANFÜHRUNG}

In Hans Georg Coenens Theorie wird deutlich gemacht, dass Metaphern, wie auch andere Formen der bildlichen Rede, Analogien voraussetzen. Hier ist die Unterscheidung von trivialen und nicht-trivialen Analogien von besonderer Relevanz. Die bildliche Rede wird ausschließlich, gleichgültig ob sie konventionell ist oder nicht, auf der Grundlage nicht-trivialer Analogien realisiert. Nicht-triviale Analogien „beruhen auf Gemeinsamkeiten, die nicht schon aus den denküblichen Klassifikationen der Analogiepartner oder aus dem semantischen Paradigma ihrer Bezeichnungen hervorgehen“ (2002, 2). Die Grundlage bildlicher Rede ist die ,in der Analogie vollzogene Umordnung der Gegenstandswelt durch Verwendung ungewohnter Klassifikationskriterien“ (12).

Seit dem 19. Jahrhundert gilt die Analogie, so Coenen, als „Triebkraft der Sprachentwicklung" (14). Im Grundprinzip geht es in Coenens Untersuchungen um die Aufdeckung des Zusammenhanges zwischen analogischer Struktur und ihrer sprachlichen Manifestation und um die Freilegung der Analogiewurzeln und ihre Darstellung als Bildfeld ${ }^{4}$ wie auch um die Bestimmung ihrer Funktion und ihrer Manifestation im Text (7). Die Analogie ist für die Aufstellung einer Theorie der Metapher insofern entscheidend, da Metaphern auf Ähnlichkeiten beruhen. Der Begriff Ähnlichkeit der herkömmlichen Rhetorik wird von Coenen durch den Analogiebegriff gleichsam ersetzt. Dies geschieht, weil der Analogiebegriff in der herkömmlichen Rhetorik seiner Meinung nach zu eng gefasst wurde. Um den Zusammenhang zwischen Analogie und Metapher verständlich zu machen, ist es notwendig, herauszustellen, dass diese Begriffe unterschiedliche Aggregatszustände darstellen: „Analogie ist eine Beziehung zwischen zwei Beschreibungsgegenständen, Metapher dagegen ein besonderes Verfahren der Wortbenutzung" (45). Metaphern sind also nicht das einzige Resultat analogischer Prozessierung.

Analogien sind bekanntlich Grundlage für Klassifizierungen, Kategorisierungen und Hierarchisierungen, also Ordnungsbildungen unterschiedlicher Art, doch kann das Verhältnis der Analogie zur Metapher nicht über eine Transformationsregel oder einen Algorithmus bestimmt werden, „dessen Anwendung die Merkmale der eigentlichen Bedeutung in die der metaphorischen überführt" (63). Die Unterscheidbarkeit von eigentlicher und metaphorischer Bedeutung wird dadurch schwierig und es bleibt oft fraglich, ob ein Begriff einen Gegenstand dank seiner eigentlichen oder vielmehr dank seiner metaphorischen Bedeutung beschreibt. ${ }^{5}$ Metaphern setzen bestimmte Unterscheidungskriterien im Sprachsystem außer Kraft, die für den eigentlichen Anwendungsbereich ausschlaggebend sind. Dadurch kommt es zu einer „Neuordnung des Gegenstandsbereichs“ und die Metapher „entwirft für ein altbe- 
kanntes Gebiet einen neuen Begriffsraster [...]“ (67-68). Dabei ist aber auch mit unkontrollierten Merkmalübertragungen zu rechnen.

Die metaphorische Bedeutung rückt zwei wesentliche Fragen in den Fokus. Erstens: „Welcher Art ist der beschriebene Gegenstand?" und zweitens: „Zu welchen anderen Gegenständen steht der beschriebene in Analogie?" (76). Im Anschluss an Coenen setzen wir voraus, dass in einem Text „eine Vielzahl von Bildern und Metaphern aus einer kleinen Zahl von Analogiewurzeln hervorwachsen“ kann (114). Auch geht er davon aus, dass es zu Erweiterungen von Analogiewurzeln kommen kann, zu einem Vorgang, bei dem eine Analogie gleichsam zu wuchern beginnt. Solche Wucherungen lassen sich auch (oder gerade) in text- und diskursübergreifender Dimension vermuten und wir setzen mit Coenen voraus, dass „Zwischen verschiedenen Analogien [...] ein systematischer Zusammenhang bestehen [kann]“ (181). Solche „paradigmatisch verwandte[n] Analogien können natürlich auch in einem gemeinsamen Kontext vorkommen“" (181). Unser Kontext ist jener der Medizin, genauer der Transplantationsmedizin. Dieser kommt jedoch in doppelter Brechung als Interdiskurs zum Tragen.

\section{DER TRANSPLANTATIONSMEDIZINISCHE FACHDISKURS IN DAVID WAGNERS BUCH}

Im Kontext der literarischen Darstellungen von Erfahrungen von Kranken und ihren Krankheiten zählt Wagners Buch zu den sog. Autopathographien ${ }^{6}$, also zu autoreflexiven Texten mit stark therapeutischer Funktion. Die deutsche Literaturwissenschaftlerin Irmela Marei Krüger-Fürhoff führt zu diesem Punkt aus: „das gemeinsame Credo von Literaturwissenschaft, Medizinsoziologie und Ethnologie [lautet], dass das [...] Erzählen einer Lebensgeschichte im Kontext von Krankheitserfahrungen eine kompensatorische, ja heilsame Funktion besitzt“ (2012, 79). Das ausschlaggebende Moment an Wagners Werk ist, dass es im Zusammenhang mit den Entwicklungen der Transplantationsmedizin steht, eines Diskurses also, der seit den „1980er Jahren medizinisch und gesellschaftlich zunehmend zum Alltag [...] gehör[t] “ (Krüger-Fürhoff 2012, 18). Diese Feststellung ändert jedoch nichts an der Tatsache, dass die Transplantation nach wie vor als etwas gilt, was sich mit dem Bild von der Natur des Menschen nicht gänzlich verträgt. Diese angenommene und ggf. vermeintliche Unverträglichkeit birgt mehrere Implikationen, die wir im Folgenden auflösen wollen und die in Wagners Buch mehr oder weniger deutlich reflektiert werden.

Zunächst einmal ist es die Identität im Sinne der individuellen Integrität, die durch die Einpflanzung eines fremden Organs strittig wird. Wagner greift diese Frage explizit auf, hinter der die Idee steckt, dass es keine Gleichheit, keine Identität mit etwas Anderem gibt. Fremdes soll jedoch in der Transplantation zum Eigenen werden und durch diese Art von Modulation relativiert sich die Grenze zwischen Selbst und Nicht-Selbst zwangsläufig, die zunächst nach der Logik der Opposition Entweder-Oder ausgerichtet war, nunmehr aber nach der Logik von Sowohl-AlsAuch eine „Hybridität von Identität“ (Krüger-Fürhoff 2012, 330) hergestellt werden soll. Die Begriffe Identität und Individualität werden dadurch porös. Das Individuum heißt entsprechend schon bei Ludwig Feuerbach der Einzelne, der sich „nur über 
die Schranken seiner Individualität erheben, aber nicht über die Gesetze, die positiven Wesensbestimmungen seiner Gattung" (1841, 369).7 Durch die Aufhebung der Individualität im Sinne der Identität des Einzelnen mit sich selbst und durch die Schaffung der hybriden Identität kommt es zur Erfahrung der Vervielfältigung, die auch Wagner deutlich reflektiert: „Ich bin ein zusammengesetzter neuer Mensch, ergänzt und verbessert, eine Chimäre, ein Hybrid, ein Replikant beinah" $(2013,163)$.

Krüger-Fürhoff macht darauf aufmerksam, dass die Transplantationsmedizin hinsichtlich der kulturellen Verträglichkeit nicht unumstritten ist $(2012,19)$, was zur Folge hat, dass Artikulationsmöglichkeiten für diverse mit der Transplantation verbundene Emotionen wie Ängste oder für kritische Hinterfragung bestimmter Konzepte wie das des Hirntodes forciert werden, da diese Möglichkeiten im Fachdiskurs oft nicht gegeben sind oder wenn, dann nur in einem sehr eingeschränkten Rahmen. Darin erkennen wir auch grundsätzlich den Sinn von Interdiskursen. Die Literatur als Interdiskurs kann das Individuell-Existentielle der Sichtweise des Patienten an gesamtgesellschaftlich relevante Fragestellungen knüpfen und auf diese Weise den Spezialdiskurs der naturwissenschaftlich analytischen Medizin in Form eines kritischen Gegendiskurses flankieren. Die Spezialdiskurse oktroyieren den Autopathographien ihrerseits bestimmte Erzählschemata und Metaphoriken auf.

Das Voraussetzungssystem des Werkes von Wagner ist medizinhistorisch und gesellschaftspolitisch einigermaßen klar verortet. Der Transplantationsskandal von 2010 bis 2012 in Deutschland liegt erst wenige Monate vor dem Erscheinungsdatum des Buches zurück. ${ }^{8}$ Die öffentlich geführte Debatte, die erst nach der Veröffentlichung des Buches in Form von rechtlichen Konsequenzen eine natürliche Fortsetzung findet, geht als paratextuelle Rahmung in die poetische Umsetzung des Themas ein und gestaltet das Voraussetzungssystem des Werkes in Form von zusätzlichen Rezeptionskontexten nachträglich.

Darüber hinaus wird das persönlich-authentische Erzählen durch Zitate gerahmt, durch Texte also, die darauf hinweisen, dass es sich dabei um nicht persönlich erlebte Erfahrungen, dennoch aber um stattgefundene Ereignisse handelt. Die eigene Erzählung wird durch die uneigene Rede förmlich kontaminiert. Hier verzahnen sich zwei wichtige Phänomene des Literatursystems: die Interdiskursivität und die Intertextualität. Die gegenseitige Bedingtheit von Interdiskursivität und Intertextualität wird bei Wagner hauptsächlich durch den Einsatz von Zitaten aus fremden, vornämlich Fachtexten realisiert. So erfolgt z. B. die Beschreibung der Hepatektomie, also der vollständigen Freilegung und Herauslösung der Leber nach einem medizinischen Lehrbuch $(2013,119)$.

Wagner bringt aber auch medizinhistorisches Wissen ins Spiel. So heißt es: „Die Leber, lese ich weiter, war lange ein geheimnisvolles Organ. [...] [D]ie Leber sei das Zentrum der Körpergeister, der Ort, dem die Körpertemperatur entspringt sowie die Quelle des Blutes" (43). Und der Autor gibt auch Beispiele aus der Antike bzw. aus der Mythologie, wo die Leber eine Rolle spielt. In diesem Fall können wir vom Wissen der Mythen sprechen. Die Geschichte von Zeus und Prometheus und die Entnahme und der Verzehr der Leber von getöteten Gladiatoren werden in die Erzählinhalte eingearbeitet. Krüger-Fürhoff macht in diesem Zusammen- 
hang aber deutlich, dass „das Zitat als Fremdkörper niemals vollständig ,einverleibt werden kann", sondern den ihn aufnehmenden Text "ebenso bestätigt wie bedroht" $(2012,55)$. Die Fremdheit (Unintegrierbarkeit) des Zitats als Fremdkörper wird bei Wagner dadurch hervorgehoben, dass es stets gesondert und kursiv abgedruckt wird.

Der eigenen Erzählung werden hier zwei Arten von fremder Rede eingepflanzt: es sind dies außer den erwähnten Zitaten aus medizinischen Fachbüchern auch noch Auszüge aus Arztberichten. Beide Arten der eingepflanzten Fremdrede heben hervor, dass eben diese darin artikulierten Sachverhalte aus der Perspektive des Patienten nicht vermittelbar sind. Wirksam wird hier die zweigliedrige geometrische Analogie von Text/Körper = Zitat/Transplantat. Diese analogische Struktur geht laut Uwe Wirth aber auch nach Krüger-Fürhoff auf Ideen von Antoine Compagnon zurück. In seinem Buch La seconde main: Ou, Le travail de la citation beschreibe Compagnon das Zitat als einen Fremdkörper, bei dem die Integration ,in den neuen Kontext den gleichen Gesetzen unterliegt wie das Spenderorgan bei einer Organtransplantation“ (Wirth 2019, 20). ${ }^{9}$ Wagner pflanzt jedoch auch Stellen aus seinen früheren Werken in seine Erzählung ein. Dies geschieht nach Compagnons Beschreibungslogik der Zweitverwertung, wenn man so will, im Sinne einer „Autotransplantation“. Die Voraussetzungen bleiben jedoch die gleichen: „neue Organe sind immer gebrauchte Organe“ (Wagner 2013, 87). Die Analogisierung der Verpflanzung des Zitats in einen neuen Textkörper beruht also auf derselben Logik wie die Transplantation eines fremden Organs in einen neuen biologischen Körperkontext. Über die Annahme bzw. die Abstoßung entscheidet der Grad der Integrierbarkeit dieses Fremdkörpers. Je unintegrierbarer ein Fremdkörper, umso mehr müssen die Abwehrkräfte des Organismus geschwächt werden. Die auf diese erzwungene Weise entstandene „Einheit", der Fremdkörper und der neue Kontext, sind dazu angehalten, sich zu konfigurieren (sich anzupassen und sich neu auszurichten), und zwar dahingehend, dass sich Fremdes und Eigenes im chimärischen Kräftefeld wechselseitig aufheben. Dieses Denkmodell macht schließlich auch das Konzept der Interdiskursivität plausibel. ${ }^{10}$ In diesem transgressiven Eigenzustand ist das entgrenzte bzw. aus dem Eigenen herausgetretene Individuum, das mit dem ihm eingepflanzten Organ nunmehr klarzukommen hat, indem die Abwehrkräfte des empfangenden Organismus supprimiert werden, auf fremde Rede und Daten angewiesen. In diesem Sinne ist Wagners Analogie „Ich bin meine Krankenakte, ich bin die Kurve meiner Werte [...]“ (280) interpretatorisch keine besondere Herausforderung.

Bei der grundlegenden Frage: „Was ist ein Organ?“ begibt sich Wagner wiederum in fremde Gewässer, genauer in die Geschichte der Philosophie. Er wird bei Thomas von Aquin fündig, der Organe und Instrumente unterschied. Und bei Schelling findet er eine passende Definition von Leben, das er als ein „Zusammenspiel mehrerer Organe“ erklärt (Wagner 2013, 198). Und Wagner fügt ein entsprechendes Zitat aus dem Buch der Kulturwisenschaftlerin und Spezialistin in Medical Humanities Katrin Solhdju ein: „Leben ist die hybride Versammlung verschiedener Organe, gemeinschaftliche Praxis, ein Konzert, in dem jedes einzelne Organ Interesse am Überleben hat" (199). Die Analogisierung des Lebens als Konzert, bei dem mehrere Instrumente/Organe miteinander im Einklang stehen ${ }^{11}$, ist philosophiegeschichtlich 
abgesichert und wird von Wagner für die Darstellung von Eigenzuständen eingesetzt: ,ich [kann] die pharmakologische Symphonie meiner Medikamente in mir rauschen hören" (38). Wir finden in Leben auch deutliche Hinweise auf den Widerstreit des botanischen und des überkommenen mechanistischen Körperkonzepts im Kontext der Transplantationsmedizin: „Von Verpflanzung wird heute kaum noch gesprochen [...]. In der älteren Literatur ist von harvesting, vom Ernten, die Rede, ein Wort, das mich erschreckt" (166-167).

Im Unterschied zu Pathographien, in denen gesellschaftliche und gesundheitliche Zustände analogisiert werden (Thomas Bernhard, Fritz Zorn, Christoph Schlingensief etc.) und wo herausgestellt wird, dass gesellschaftliche Konstellationen direkt Pathologien verursachen, bzw. dass Krankheiten eine unmittelbare Reaktion auf ungünstige und kritikwürdige gesellschaftliche Strukturen darstellen, wird in Wagners Text die Krankheit als Teil des Einzigen, als eine Ingredienz des Lebens und des Menschenbildes geschildert. Ein wesentliches Merkmal dieser Texte ist, dass sie die jeweilige Sparte der Medizin sachkundig ausleuchten und über den medizinischen Forschungsstand reflektieren, Fachtexte in die Erzählstruktur integrieren und nicht selten, wie in Wagners Fall, diese auch sach- und fachgerecht zitieren. Dadurch koppelt dieses Genre die introspektive Authentizität an den medizinischen Spezialdiskurs, ohne jedoch potentielle Dissonanzen zwischen dem (sich selbst) erlebenden und hinterfragenden Subjekt und dem zu behandelnden Objekt oder die eventuelle Fragwürdigkeit der biologischen Grenzen zwischen dem Eigenen und dem Fremden ausblenden zu wollen/können. Gerade diese Grenzen werden von der (bio)technologisch fundierten Transplantationsmedizin ein Stück weit durchlässig gemacht. Die Transplantation an sich ist geradezu ein Akt der Infragestellung biologischer Grenzen. Die imaginierten Gespräche mit dem/der abwesenden Spender/Spenderin und das Gefühl der Intimität bzw. sogar einer Liebesbeziehung und gleichzeitig Verschwisterung, die sich aus dieser Erfahrung ergibt, legen ein deutliches Zeugnis davon ab, dass hier vielmehr ein Zusammenwachsen stattfindet anstelle eines Empfanges oder Austausches. Die wahrgenommene Eigenhybridität färbt natürlich auch auf die reflektierende Wahrnehmung des Spenders und des Spenderorgans ab: Der Spender ist wahlweise weiblich und ein Zwitterwesen: „Sie lacht und schaut sehr ernst dabei, sie ist blond und hat kohlrabenschwarzes Haar" (Wagner 2013, 133).

Die medizinisch bekannte aber dennoch nach wie vor mysteriöse Problematik des Chimärismus verbindet Literatur und Medizin in besonderer Weise - ein Phänomen, das stellvertretend für alle anderen Phänomene die engen Grenzen des rational (wissenschaftlich) Deutbaren gleichsam sprengt. Diese diskursiven Leerstellen werden über mehr oder weniger bewährte Analogisierungsstrategien ausgefüllt.

Die Welt der Medizin wird in Wagners Text als ein eigenes Universum bzw. als Meer konzeptualisiert, in dem das Krankenbett als Raumschiff bzw. als Schiff und die Krankheit als Reise metaphorisiert werden. Die dahinterliegende Analogie bleibt wurzelgleich.

Beispiel 1: „Sehr viele Apparate um mich herum, Kabel, drei oder vier Monitore, ich höre ein Piepen. Kommandozentrale? Mir gefällt mein Raumschiff, ich bin leicht, schwerelos, ich kann fliegen“ (6). „[I]ch schwebe [...] - erst nach Minuten, Stunden oder Tagen muß ich in meine Haut zurück, in dieses Bett“ (17). 
Beispiel 2: „Dieses Bett ist wirklich mein Raumschiff und ich bin unterwegs zum Mars“ (18).

Beispiel 3: „Ich liege in einem riesigen Raumschiff, die Schwestern sind gutprogrammierte Pflegeroboter" (80).

In allen drei Beispielen wird eine Ähnlichkeit des Patienten in seinem Krankenbett mit einem Astronauten in einer Raumkapsel zum Ausdruck gebracht. Beide sind unterwegs jeweils in einem System höherer Ordnung: Der Patient in seinem Krankenbett im System der medizinischen Einrichtung und der Astronaut in seiner Raumkapsel im Weltall. Diese Analogien ordnen sich zu einem fünfstelligen Bildfeld:

\begin{tabular}{|l|l|l|l|l|l|}
\hline & 1 & \multicolumn{1}{|c|}{2} & 3 & \multicolumn{1}{c|}{5} \\
\hline A & Astronaut & Schwerelosigkeit & Raumkapsel & Weltraum & $\begin{array}{l}\text { Zielort: } \\
\text { ferner Planet }\end{array}$ \\
\hline B & Patient & $\begin{array}{l}\text { Leichtigkeit/ } \\
\text { Schwebezustand }\end{array}$ & Krankenbett & Klinikum & $\begin{array}{l}\text { Zielort: } \\
\text { Genesung }\end{array}$ \\
\hline
\end{tabular}

Strukturformel: (1) bewegt sich in der Art von (2) in einem geschützten Gehäuse (3) in der Gesamtheit der Elemente eines Systems höherer Ordnung (4) auf ein Ziel (5) hin.

Das Besondere an diesen wurzelgleichen Analogien ist, dass das Krankenbett nicht nur mit der Raumkapsel eines Astronauten analogisiert wird, sondern dass beides mit der schützenden Haut des Individuums identifiziert wird. Hier klingt die Vorstellung der Verschmelzung der Kapsel mit dem Ich an - eine Vorstellung, die bei Oswald Wiener in seinem Konzept des Bio-Adapters eindrücklich realisiert wurde.

In diesem Kontext kommt die Konvention der Metaphorik des Lebens als Reise zum Tragen. Mit einer Zeile aus Kleists Drama Prinz Friedrich von Homburg wird diese Konvention auf direktem Wege herbeizitiert: „[D]as Leben nennt der Derwisch eine Reise" (35). In der analogischen Struktur wird auch die Autoreflexivität der menschlichen Existenz nach diesem bekannten Muster umgesetzt. Das reflektierend lokomotierende Subjekt nimmt sich selbst dabei als eine labyrinthartige Gegend wahr: „[Ich] denke in mir herum und einmal quer hindurch. Und verirre mich in $\mathrm{mir}^{\text {" }}(64)$.

Angesichts der existenziellen Problematik des Buches werden nach diesem Modell auch weitere Erfahrungen konzeptualisiert. Hier lassen sich also zwei Unterarten einer Globalanalogie ausmachen: Die eine, wie oben dargestellt, nimmt als erstes Glied der Analogiemenge ein sich im Weltraum bewegendes Raumschiff zur Grundlage der Analogiebildung. Bei der anderen, wie im Folgenden gezeigt wird, ist es ein Schiff auf dem Meer. Der Beschreibungsinhalt, der auf geordneten Gegenstandsmengen beruht, ist in beiden Fällen identisch:

Beispiel 1: „ein automatisches Blutdruckmessgerät [...] Hört sich an, als puste jemand in eine Luftmatratze. Auf dieser Luftmatratze treibe ich aufs Meer" (15).

Beispiel 2: „Ich schlafe in einer Außenkabine, in der Bordwand ein Bullauge, ich sehe Wasser, viel Wasser, manchmal zieht eine Insel vorbei, ein U-Boot taucht auf, ein Eisberg treibt 
dahin oder ein einsamer Schwimmer, der fast schon aufgegeben hat. Das muß die Vergangenheit sein. Ich habe mich eingeschifft, bin an Bord [...] die Krankheit ist eine große Reise, le grand tour, einmal in die Unterwelt und vielleicht zurück“ (26).

Beispiel 3: „Ich habe abgelegt, ich treibe auf meinem Floß, ich bin meine eigene Insel, drifte über meinen Ozean [...] Kreuzfahrt durchs Ich und dieses Krankenhaus" (69).

Beispiel 4: „Du [...] wir liegen zusammen auf diesem Floß, wir treiben zusammen über dieses Meer" (282).

In allen vier Beispielen wird eine Ähnlichkeit des Patienten in seinem Krankenbett mit einem Schiffspassagier in einem Wasserfahrzeug (bzw. einer Schiffskabine) zum Ausdruck gebracht. Auch in diesem Fall sind beide jeweils auf der Reise in einem System höherer Ordnung. Diese Analogien ordnen sich, da sie mit der oben dargestellten Analogie wurzelgleich sind, zu einem fünfstelligen Bildfeld:

\begin{tabular}{|l|l|l|l|l|l|}
\hline & \multicolumn{1}{|c|}{1} & \multicolumn{1}{c|}{2} & \multicolumn{1}{c|}{3} & \multicolumn{1}{c|}{4} \\
\hline A & Schiffspassagier & $\begin{array}{l}\text { Treiben auf der } \\
\text { Wasseroberfläche }\end{array}$ & Wasserfahrzeug & Meer & $\ldots$ \\
\hline B & Patient & $\begin{array}{l}\text { Leichtigkeit/ } \\
\text { Schwebezustand }\end{array}$ & Krankenbett & Klinikum & $\ldots$ \\
\hline
\end{tabular}

Strukturformel: (1) bewegt sich in der Art von (2) in einer Vorrichtung (3) im Element eines Systems höherer Ordnung (4) auf ein Ziel (5) hin.

Auch in diesem Fall können wir beobachten, dass das Krankenbett nicht nur mit der Schiffskabine eines Passagiers analogisiert wird, sondern dass beides wiederum mit der Identifizierung dieser mit dem Individuum einhergeht („,ich bin meine eigene Insel“). Die Idee hinter dem wienerschen Konzept des Bio-Adapters kommt auch hier erneut zum Tragen. Das Fundament dieser wurzelgleichen Analogien ist, dass sie in die metaphorische Struktur der „Krankheit als Reise“ eingebettet ist, die wiederum das Muster der Metapher „Leben als Reise“ kopiert. Auch die Struktur dieser Globalanalogie eröffnet die Möglichkeit für den Analogieschluss, dass das Ziel die Genesung darstellt. Dieses Ziel findet in diesem Fall jedoch keine sprachliche Umsetzung, ist aber naheliegend und daher der analogischen Struktur als inhärent anzusehen.

Dem gegenüber überwuchert der Text gleichsam mit Imaginationen und Assoziationen, die sich um die Leerstelle des unbekannten Organspenders ranken. Bekanntschaft und Vertrautheit werden entsprechend mit Hilfe von bewährten Konzepten der Verwandtschaft konstruiert: „Plötzlich wird mir klar, daß du [...] auch in einigen anderen Menschen weiterlebst. Ich habe dich gar nicht für mich allein, Liebste [...] ich habe Transplantationsgeschwister“ (186). Die Phänomene der Hybridität und des Chimärismus sind diesen Imaginationen in Form von als komplementär dargestellten Gegensatzpaaren eingeschrieben. Das Phänomen des Chimärismus (163), das im medizinischen Fachdiskurs im Kontext der Erforschung von Abstoßungsreaktionen als belegt gilt, findet in Wagners Text unter mehrfacher Berufung vor allem auf Forschungserfolge von Thomas E. Starzl (156-157) interdiskursive Umsetzung. 
Es scheint auch, dass das Verständnis der Organverpflanzung davon abhängig ist, wie das Phänomen des Chimärismus kommuniziert wird, denn die Transplantationsmedizin macht klar, dass das eingepflanzte Organ und der Organempfänger sich durch einen reziproken adaptiven Zellenaustausch aufeinander einstimmen, d. h. dass nicht nur das Organ Zellen des Organismus aufnimmt, sondern dass auch im Organismus Zellen des eingepflanzten Organs festgestellt werden konnten. Diese neue chimärische Seinsart wird vom Ich-Erzähler aufgegriffen und um die Frage erweitert, inwiefern der bio-chemische Chimärismus mit Adaptionen der Persönlichkeit zu tun haben könnte. Die sich daran anschließenden Zweifel an der Ursprünglichkeit des eigenen Empfindens sind nur noch ein konsequentes und nachvollziehbares Folgeglied dieser Gedankenkette.

\section{DOMÄNENÜBERGREIFENDE PROJEKTIONEN, HYBRIDITÄT UND CHIMÄRISMUS}

Uwe Wirth und Ottmar Ette machen darauf aufmerksam, dass das bekannte Konzept der Transplantation „aus einem Geflecht von metaphorischen Überblendungen besteht, die botanisches Erfahrungswissen auf medizinische Fragestellungen überträgt“ $(2019,2)$. Auf dieses Konzept antwortet auch Wagner mit der Bemerkung: „Vielleicht sollte ich nur denken, mir sei ein Ersatzteil eingebaut worden wie einem Auto. Auf diese Weise wäre ich die botanische Metaphorik los" $(2013,167)$. Darüber hinaus machen Wirth und Ette unter Berufung auf Friedrich Schleiermacher und Walter Benjamin klar, dass die Metapher der floristischen Einflussnahme durch Verpflanzung für „Übersetzungen aller Art“ $(2019,3)$ stehe. Dies inkludiert die Überlegung, dass die Medizin (wie auch schon die Übersetzungstheorie) das floristische (und ursprünglich die Pfropftechnik bezeichnende) Konzept, das sich im „19. Jahrhundert als Begriff der chirurgischen Transplantation im heutigen Sinne“ (Wirth 2019, 11) durchgesetzt hat, reintegriert. ${ }^{13}$ Prozesse der Begriffs- wie auch der Metaphernbildung unterliegen somit denselben Prinzipien und diese bedingen einander umso mehr, wenn es sich, wie im Falle des Konzepts der Transplantation, um nomadisierende Konzepte handelt. Das Konzept der Pfropfung wird dabei gleichsam mit übernommen. Und Wirth führt weiter aus: „immerhin hilft die epistemische Metapher der Pfropfung, auf der Unterlage eines alten Repertoirs [sic] von Terminologien, ein Bewusstsein für die neuen Probleme zu entwickeln“ (12).

Analogien zwischen pflanzlichen, tierischen, geologischen und oft eben auch technisch-maschinellen Domänen sind hinsichtlich der gegenseitigen Beeinflussung von begrifflichen und metaphorischen Konzepten ausgesprochen fruchtbar und all diese Übertragungen lassen sich auf das nomadisierende Kernkonzept der Organtransplantation herunterbrechen.

Wagner bemüht in diesem Sinne die Maschinen-Metaphorik dementsprechend, obwohl er ihr gegenüber nicht unkritisch eingestellt ist:

Beispiel 1: „Und du, Organ, gehörst jetzt wirklich mir? [...] Bin ich bloß der Behälter, der Apparat, der dich am Leben erhält?" (178).

In diesem Beispiel handelt es sich um eine Metapher mit einer einstelligen Analogie. Die Bedingung für die Metaphernbildung ist, dass die Analogie nicht trivial ist. 
Das Besondere an diesem Beispiel ist, dass das Wurzelprädikat (erhält das Organ am Leben) vom Autor gleichsam ausformuliert wird. Die Interpretation der Metapher wird gleich mitgeliefert.

Beispiel 2: „Schläuche [...] als wäre mir eine Schnittstelle eingebaut worden [...] Die Schnittstelle ist die Wunde, die Wunde ist die Schnittstelle [...]“ (156).

Hier wird eine Ähnlichkeit des Patienten mit einem Datenverarbeitungssystem zum Ausdruck gebracht. Diese Analogie ordnet sich zu einem dreistelligen Bildfeld:

\begin{tabular}{|l|l|l|l|l|}
\hline & \multicolumn{1}{|c|}{1} & \multicolumn{1}{c|}{2} & \multicolumn{1}{c|}{3} & \multicolumn{1}{c|}{4} \\
\hline A & Datenverarbeitungssystem & Datenkabel & Schnittstelle & (anderes System) \\
\hline B & Patient & Schläuche & Wunde & (anderes System) \\
\hline
\end{tabular}

Strukturformel: (1) wird über (2) an der (3) mit (4) verbunden.

Aus der Struktur dieser Analogie ergibt sich die Möglichkeit für den Analogieschluss, dass (1) an ein anderes System angeschlossen ist oder angeschlossen werden kann. Diese Stelle findet in diesem Fall keine sprachliche Umsetzung, ist aber in der analogischen Struktur angelegt und für die Interpretation der Metapher „die Wunde ist eine Schnittstelle" entscheidend. Diese Metapher ist nur ein Teil einer größeren Analogiewurzel.

Auf der anderen Seite greift Wagner auf die bewährte Tier-Metaphorik zurück. Diese bezieht er auf das betreffende Organ (Wal), die Operationsnarbe (Insekt) und sich selbst (Ente). Zuletzt wird die Metapher des fressenden Krebses in Anschlag gebracht:

Beispiel 1: „Die Leber, mein weißer Wal, liegt, groß und ruhig und rundgeschwollen [...]“ (40).

Hier handelt es sich um eine Metapher mit einer einstelligen Analogie. Auch in diesem Fall wird das Wurzelprädikat (liegt groß und massig) vom Autor ausformuliert.

Beispiel 2: „dieses Etwas, das sich unterhalb meines Brustkorbs in die Haut gekrallt hat, sieht aus wie ein riesiges schwarzes Insekt“"(155-156).

In diesem Fall handelt es sich wiederum um eine Metapher mit einer einstelligen Analogie. Das Wurzelprädikat (krallt sich in die Haut, so dass ein wurstförmiges Gebilde entsteht) wird vom Autor als Interpretationsansatz vorgegeben.

Beispiel 3: „Ich schwebe über einer Stadt [...] Ich habe ja Flügel [...] ich bin ein Vogel, ich bin eine Ente, ich bin die Ente im Zwischenreich [...]“ (131).

In Beispiel 3 wird eine Ähnlichkeit des Patienten mit einem Vogel zum Ausdruck gebracht. Diese Analogie ordnet sich zu einem dreistelligen Bildfeld:

\begin{tabular}{|l|l|l|l|}
\hline & \multicolumn{1}{|c|}{1} & \multicolumn{1}{|c|}{2} & \multicolumn{1}{c|}{3} \\
\hline A & Vogel/Ente & Flügel & schweben im Zwischenreich \\
\hline B & Patient & Flügelhemd & schweben im Zwischenreich \\
\hline
\end{tabular}

Strukturformel: (1) verfügt über (2) was ihn zu (3) zu befähigen scheint. 
In der Struktur dieser Analogie sind praktisch alle Elemente der Analogiemenge ausformuliert bis auf den Analogieschluss, der sich aus der Logik des Beschreibungsinhalts und entsprechend der Wortbedeutung Zwischenreich ${ }^{14}$ ergibt.

Auch die geologische Metaphorik wird von Wagner in einigen Fällen aufgegriffen. Die Zieldomänen sind dabei mal die Operationswunde und mal die Müdigkeit:

Beispiel 1: „die Hand geht auf Expedition ins Ungewisse [...] nach Süden, Richtung Nabeläquator [...] den ersten Ausläufer des Wurstgebirges [...] ein doppelter Karpatenbogen zieht sich über meinen Bauch“" (181-182).

Hier wird eine Ähnlichkeit der Operationsnarbe mit einem geometrischen/ mathematischen (Krümmung) und mit einem geographischen Gebilde (Erhebung im Gelände) zum Ausdruck gebracht. In diesem Fall haben wir es mit einer Metapher zu tun, die sich aus einer zweistelligen Analogie ergibt. Das Wurzelprädikat beruht also auf der Ähnlichkeit der Wunde mit einem gekrümmten Kurvenstück und einem geografischen Gebilde.

Beispiel 2: „Die Müdigkeit ist ein Berg, den ich hinabrolle, ist ein tiefes Tal, ist eine Ebene, weit und leer und karst zugleich. Sie hat die amorphe Topographie eines unbekannten Planeten“ (248).

In diesem Beispiel wird die geographische Metaphorik mehrfach ausgebeutet. In allen vier Fällen der Analogisierung (Berg, Tal, Ebene, Karstlandschaft) haben wir es mit Metaphern zu tun, die sich aus einstelligen Analogien ergeben.

\section{SCHLUSSBEMERKUNG}

Die dargestellten domänenübergreifenden Projektionen führen zu Vermengungen von Konzepten bzw. zu Konzeptualisierungen von Vermengungen (Hybridisierung), wie sie bei Wagner eindrücklich thematisiert werden. Dieses konzeptuelle Hinüberwandern mündet in die Vorstellung des Menschen als Ersatzteillager, und es heißt bei Wagner entsprechend: „Vielleicht sollte ich besser denken, mir sei bloß ein Ersatzteil eingebaut worden. Wie einem Auto“ (137).

Die von Wagner angedachte Untragbarkeit der Maschinen-Metaphorik wird richtiggehend in Bezug auf die Vermengung von Zelltypen im Kontext des Chimärismus-Phänomens für das globale Verständnis des Buches relevant, zumal auf diese Weise deutlich wird, wie sich die verschiedenen Konzepte des Organs (physiologisch-biophysikalisch vs. instrumental-maschinell) gegenseitig voraussetzen. Und auch in diesem Punkt ist Wagner durch Fachdiskurse sensibilisiert.

\section{ANMERKUNGEN}

1 Nicht zuletzt sollen diese Einsichten im medizinischen Bereich auf Resonanz stoßen und Alexander Mitscherlich zur Formulierung der wichtigsten Aufgabe der psychosomatischen Medizin bewegen, die darin besteht, „ein ausreichendes Wissen von den Regelungsvorgängen im Erlebnisbereich und seiner Entwicklung zu sammeln“([1967] 1975, 81).

2 Obwohl es ihm nicht darum geht, Erlebnispsychologie zu betreiben, hält Bühler ganz im Sinne seiner Sprachtheorie fest, dass im Erlebnis oft „eine Sphären-Zweiheit“ nachzuweisen sei (1934/65, 343). 
3 Hermann Paul war derjenige, der die Analogie als einen wesentlichen Faktor im Sprachleben untersucht hatte.

${ }^{4}$ Der Coenen'sche Begriff des Bildfeldes ist von jenem von Harald Weinrich zu unterscheiden. Unter diesem Begriff versteht Coenen eine Analogiewurzel, die auf eine bestimmte Art und Weise zur Darstellung gelangt. So führte Coenen aus: „Ein Bildfeld ist die Darstellung einer Gegenstandsmenge, die sich in - mindesten zwei - geordnete, gleich große und gleich strukturierte Untermengen, die ,Bildfeldbereiche', gliedert“ (39). Ein Bildfeld ist somit ein Schema, durch das eine Analogiesetzung schematisch erklärt wird.

5 Weiter unten wird diese Problematik in Bezug auf nomadisierende Konzepte aufgegriffen.

6 Ich verwende diesen Begriff im Sinne von Monika Kohlhage, die ihn auf die Krankheitsgeschichten von Thomas Bernhard bezog $(1987,19)$.

7 Max Stirner rekurriert auch auf Feuerbachs Auffassung vom Individuum und zitiert aus dessen Werk Das Wesen des Christentums: „Egoismus und Menschlichkeit (Humanität) müßten das Gleiche bedeuten, aber nach Feuerbach kann der Einzelne (das ,Individuum'), sich nur über die Schranken seiner Individualität erheben, aber nicht über die Gesetze, die positiven Wesensbestimmungen seiner Gattung" $(1901,186)$. Und im Anschluss daran formuliert Stirner folgenden fundamentalen Gedanken: „Allein die Gattung ist nichts, und wenn der Einzelne sich über die Schranken seiner Individualität erhebt, so ist dies vielmehr gerade Er selbst als Einzelner, er ist nur, indem er sich erhebt, er ist nur, indem er nicht bleibt, was er ist; sonst wäre er fertig, tot. Der Mensch ist nur ein Ideal, die Gattung nur ein Gedachtes“ (186).

8 Im Werk selbst wird Thomas Starzls Lieblingsschüler und Chefarzt in Essen in diesem Zusammenhang namhaft gemacht (Wagner 2013, 276).

9 Vgl. auch Ausführungen von Krüger-Fürhoff 2012, 55.

${ }^{10}$ Der Frage, wie diese Analogisierung von Zitieren und Transplantieren prozessiert wird, was genau ausgeblendet wird, sprich, welche Selektionsstrategien bei der Darstellung der Integration fremden Materials in den eigenen Körper zum Tragen kommen, werden wir uns gesondert zuwenden.

${ }^{11}$ Von concertare - zusammen verabreden, zusammen streiten (Diez 1887, 106).

${ }^{12}$ Der Schwebezustand zwischen Schlafen und Wachen.

${ }^{13}$ Vgl. dazu auch Fichtner 1968.

${ }^{14}$ Laut DUDEN: Bereich zwischen Leben und Tod (https://www.duden.de/rechtschreibung/Zwischenreich).

\section{LITERATUR}

Bühler, Karl. [1934] 1965. Sprachtheorie. Die Darstellungsfunktion der Sprache. 2. unveränderte Auflage. Stuttgart: Gustav Fischer Verlag.

Coenen, Hans Georg. 2002. Analogie und Metapher. Grundlegung einer Theorie der bildlichen Rede. Berlin - New York, NY: De Gruyter.

Diez, Friedrich. 1887. Etymologisches Wörterbuch der romanischen Sprachen. 5. Ausgabe. Bonn: Adolph Marcus.

Ette, Ottmar - Uwe Wirth, Hrsg. 2019. Kulturwissenschaftliche Konzepte der Transplantation. Berlin Boston, MA: De Gruyter.

Feuerbach, Ludwig. 1841. Das Wesen des Christentums. Leipzig: Otto Wigand.

Fichtner, Gerhard. 1968. „Das verpflanzte Mohrenbein. Zur Interpretation der Kosmas-und-Damian -Legende." Medizinhistorisches Journal 3, 2: 87-100.

Kohlhage, Monika. 1987. Das Phänomen der Krankheit im Werk von Thomas Bernhard Taschenbuch. Herzogenrath: Verlag Murken-Altrogge.

Krüger-Fürhoff, Irmela Marei. 2012. Verpflanzungsgebiete. Wissenskulturen und Poetik der Transplantation. München: Wilhelm Fink Verlag.

Mitscherlich, Alexander. [1967] 1975. Krankheit als Konflikt. Studien zur psychosomatischen Medizin 2. 6. Auflage. Frankfurt am Main: Suhrkamp. 
Stählin, Wilhelm. 1914. „Zur Psychologie und Statistik der Metaphern: Eine methodologische Untersuchung." Archiv für die gesamte Psychologie 31: 297-425.

Stirner, Max. 1901. Der Einzige und sein Eigentum. Leipzig: Verlag von Otto Wigand.

Wagner, David. 2008. „Für neue Leben.“ Merkur 62, 715: 1113-1122.

Wagner, David. 2013. Leben. Reinbek bei Hamburg: Rowohlt Verlag.

Werner, Heinz. 1919. Die Ursprünge der Metapher. Leipzig: W. Engelmann.

Wirth, Uwe. 2019. „Konzepte und Metaphern der Transplantation.“ In Kulturwissenschaftliche Konzepte der Transplantation, hrsg. von Ottmar Ette - Uwe Wirth, 9-28. Berlin, Boston: De Gruyter.

\title{
On the poietic function of analogies and metaphors in the interdiscursive perspective of transplant medicine
}

Interdiscursivity. Metaphor. Analogy. Medical specialist discourse. Organ

transplantation. Theory of figurative language. Hans Georg Coenen. David Wagner.

The subject of the analysis is the novel Leben (Life, 2013) by the German author David Wagner. In fact, although designed as a novel, the book is more precisely an autopathography, which presents a personal and authentically reflective look at the experience of one's own illness and describes the medical intervention, the surgical transplantation of a new liver. The medical specialist discourse plays an essential role in this context. Medical knowledge and personal reflections overlap like those areas of meaning from which analogies often emerge and in which metaphors have their origin. In the following, it is a matter of uncovering those areas that are combined to create analogies in order to describe their communicative function on the basis of their possible implementation in the form of metaphors (also known as between-domain analogies). Particular consideration is given to the role of the medical specialist discourse and the question of how it shapes the analogization and metaphorization of one's own experience of illness and the experience of organ transplantation. In doing so, we rely largely on the analytical model based on the theory of figurative language by Hans Georg Coenen.

\author{
Mgr. Roman Mikuláš, PhD. \\ Institut für Weltliteratur \\ Slowakische Akademie der Wissenschaften \\ Dúbravská cesta 9 \\ 84104 Bratislava \\ Slowakische Republik \\ roman.mikulas@savba.sk \\ ORCID: https://orcid.org/0000-0002-0822-2535
}

